\title{
Challenges and perspectives in combinatorial assembly of novel exopolysaccharide biosynthesis pathways
}

\author{
Anke Becker* \\ LOEWE Center for Synthetic Microbiology and Faculty of Biology, Philipps-University of Marburg, Marburg, Germany
}

Because of their rheological properties various microbial polysaccharides are applied as thickeners and viscosifiers both in food and non-food industries. A broad variety of microorganisms secrete structurally diverse exopolysaccharides (EPS) that contribute

Edited by:

Jochen Schmid,

Technische Universität München,

Germany

Reviewed by:

Dayananda Chandrappa,

University of Exeter, UK

Salim T. Islam,

CNRS - Laboratoire de Chimie

Bactérienne, France

Kathryn M. Jones,

Florida State University, USA

*Correspondence:

Anke Becker,

LOEWE Center for Synthetic

Microbiology and Faculty of Biology,

Philipps-University of Marburg,

Hans-Meerwein-Street 6,

35043 Marburg, Germany

anke.becker@synmikro.uni-

marburg.de

Specialty section:

This article was submitted to

Microbiotechnology, Ecotoxicology and Bioremediation,

a section of the journal

Frontiers in Microbiology

Received: 14 April 2015

Accepted: 22 June 2015

Published: 09 July 2015

Citation:

Becker A (2015) Challenges

and perspectives in combinatorial assembly of novel exopolysaccharide

biosynthesis pathways.

Front. Microbiol. 6:687.

doi: 10.3389/fmicb.2015.00687 to their surface attachment, protection against abiotic or biotic stress factors, and nutrient gathering. Theoretically, a massive number of EPS structures are possible through variations in monosaccharide sequences, condensation linkages and non-sugar decorations. Given the already-high diversity of EPS structures, taken together with the principal of combinatorial biosynthetic pathways, microbial polysaccharides are an attractive class of macromolecules with which to generate novel structures via synthetic biology approaches. However, previous manipulations primarily focused on increasing polysaccharide yield, with structural modifications restricted to removal of side chains or non-sugar decorations. This article outlines the biosynthetic pathways of the bacterial heteroexopolysaccharides xanthan and succinoglycan, which are used as thickening and stabilizing agents in food and non-food industries. Challenges and perspectives of combining synthetic biology approaches with directed evolution to overcome obstacles in assembly of novel EPS biosynthesis pathways are discussed.

Keywords: polysaccharide, synthetic biology, glycosyltransferase, synthase-dependent pathway, ABC transporterdependent pathway, Wzx/Wzy-dependent pathway

\section{Introduction}

A broad variety of polysaccharides are naturally produced by bacteria, fungi, algae, and plants. Bacteria are able to synthesize surface polysaccharides including lipopolysaccharides (LPS) constituting the outer leaflet of the outer membrane of Gram-negative bacteria, capsular polysaccharides (CPS) bound to the cell surface, and secreted exopolysaccharides (EPS). As the interface between the bacterial cell and the environment, surface polysaccharides play important roles in protection against abiotic or biotic stress factors, nutrient gathering, surface attachment, motility, and interactions with host immune systems (Rehm, 2009; Ullrich, 2009; Donot et al., 2012). Variations in monosaccharide composition, condensation linkages, non-sugar decorations, and molecular weight give rise to an enormous diversity of structures that contributes to their diverse biological functions. This diversity also accounts for an attractive spectrum of physical and rheological properties of microbial EPS opening up commercial applications in industrial, food and medical sectors as thickening, emulsifying, chelating, or stabilizing agents (Freitas et al., 2011). Many microbial polysaccharides have properties similar to traditionally applied gums originating 


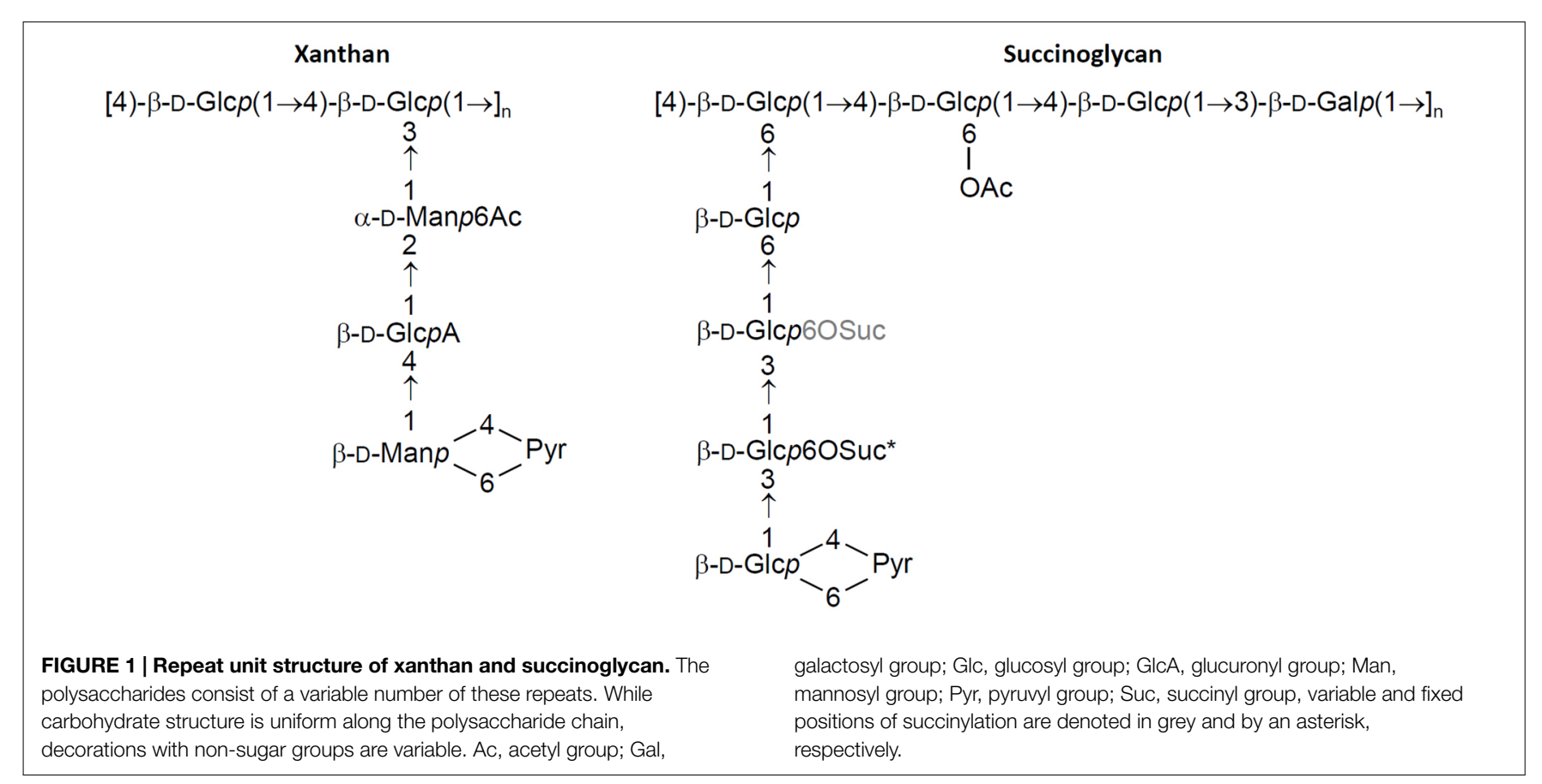

from plants or algae. Prominent examples of commercially applied microbial EPS are xanthan gum, gellan, and alginate. While xanthan is primarily used in cosmetics, food and oil industry (Becker et al., 1998), gellan and alginate are also applied in pharmacy and medicine, e.g., in wound healing, tissue engineering and drug delivery (Lee and Mooney, 2012; Osmalek et al., 2014). Furthermore, an increasing number of algal and microbial polysaccharides with novel properties are being discovered (Paniagua-Michel Jde et al., 2014).

Exopolysaccharides are either homo- or heteropolymers which are frequently decorated by non-carbohydrate substituents, such as acetyl, pyruvyl, or succinyl groups, which confer anionic properties to the polysaccharide. Heteropolymeric EPS are typically composed of identical repeat units that may only vary by the presence of decorating groups. Assembly of the repeat units to the polymer can result in branched structures.

Exopolysaccharides biosynthesis is a multistep process comprising the

(i) synthesis of nucleotide sugar precursors

(ii) synthesis of oligosaccharide repeat units or direct synthesis of the polysaccharide by successive or progressive activity of glycosyltransferases

(iii) assembly of the polysaccharide from the repeat units

(iv) export of the product

Nucleotide diphosphates (NDPs) or nucleotide monophosphates (NMPs) are the common precursors for the carbohydrate components of polysaccharide biosynthesis pathways. They serve as activated donors for the glycosyltransferase-catalyzed transfer of the sugar to a lipid carrier or a carbohydrate. Polysaccharides are assembled and exported by one of three known distinctive types of mechanisms: the synthase- (Whitney and Howell,
2013), ATP-binding cassette (ABC) transporter- (Greenfield and Whitfield, 2012; Willis and Whitfield, 2013), and Wzx/Wzydependent (Islam and Lam, 2014, and summarized by Schmid et al., 2015) pathways.

The broad range of structural diversity of secreted branched heteropolysaccharides makes their biosynthetic pathways ideal candidates for design of novel structures by synthetic biology approaches. Such polysaccharides are typically built from repeat units that are assembled by the Wzx/Wzydependent pathway. Through more detailed elucidation of this biosynthetic pathway, novel tailored EPS may eventually be generated via combinatorial strategies using an engineered modular apparatus. However, to date the most successful engineering approaches addressed improvements in the yield or production process, alterations in the degree of polymerization, removal of side chains or non-sugar substituents, or heterologous expression of EPS biosynthesis gene clusters (Rehm, 2009; Ullrich, 2009). This review outlines the well-studied biosynthetic pathways of the acidic heteroexopolysaccharides xanthan and succinoglycan applied in cosmetics, food and oil industry (Becker et al., 1998; Fink, 2003a,b; De et al., 2015; Figure 1). It discusses obstacles, perspectives, and the needs for research of molecular mechanisms operating at different steps of biosynthesis to promote synthetic biology approaches toward assembly of pathways producing novel EPS structures.

\section{Biosynthesis of Xanthan and Succinoglycan by the Wzx/Wzy-dependent pathway}

Xanthan produced by Xanthomonas campestris is composed of pentasaccharide repeat units, forming a cellulose backbone 
with trisaccharide side-chains of $[\beta$-D-Man $p-(1 \rightarrow 4)-\beta$-D-GlcpA$(1 \rightarrow 2)-\beta$-D-Man $p-(1 \rightarrow)]$ attached to alternate glucose residues in the backbone by $\alpha-1,3$ linkages (Jansson et al., 1975; Melton et al., 1976; Figure 1). The terminal mannose residues can be modified by a pyruvic acid group attached by a ketal linkage and acetyl groups often decorate as 6-O substituents the internal mannose residues. Some external mannoses carry a second 6O-acetyl substituent (Ielpi et al., 1981, 1983; Stankowski et al., 1993). Succinoglycan produced by Sinorhizobium meliloti is made of octasaccharide repeat units containing one galactose and seven glucose residues joined by $\beta-1 \rightarrow 3, \beta-1 \rightarrow 4$, and $\beta-1 \rightarrow 6$ linkages (Figure 1). The terminal glucose residue is substituted by a pyruvyl group while acetyl and succinyl groups decorate inner glucose residues (Aman et al., 1981; Reinhold et al., 1994).

Typically, genes directing synthesis, polymerization and export of a specific polysaccharide are clustered in the bacterial genome. In contrast, genes involved in the synthesis of common nucleotide sugar precursors required for the production of more than one oligo- or polysaccharide are frequently uncoupled from the specific biosynthesis gene clusters (Harding et al., 1993). However, many clusters contain additional copies of these genes or genes for the synthesis of nucleotide sugar precursors specific to the polysaccharide. In $X$. campestris and S. meliloti, the $16 \mathrm{~kb}$ gum and the $24 \mathrm{~kb}$ exo gene cluster, respectively, encode glycosyltransferases, enzymes catalyzing the addition of nonsugar decorations, and proteins involved in the terminal steps of xanthan and succinoglycan biosynthesis (Becker and Pühler, 1998; Becker et al., 1998; Figure 2). While in the succinoglycan biosynthesis gene cluster, exoB and exoN encode a UDP glucose 4-epimerase and a UDP-glucose pyrophosphorylase, respectively, the xanthan biosynthesis gene region does not encode enzymes involved in synthesis of nucleotide sugar precursors.

Both EPS are synthesized by the Wzx/Wzy-dependent pathway named after the key components involved in flipping the lipid carrier with the repeat unit from the cytoplasmic to the periplasmic face of the inner membrane (Wzx) and assembly of the repeat units to the polymer (Wzy). Repeat units are synthesized on a C55-undecaprenol phosphate (und-P) lipid carrier located in the inner leaflet of the cytoplasmic membrane by the sequential activity of glycosyltransferases as has been revealed by the accumulation of lipid-carrier bound oligosaccharide intermediates in glycosyltransferase mutants (Ielpi et al., 1993; Reuber and Walker, 1993). Initiation of the repeat unit synthesis is catalyzed by a member of the polyisoprenylphosphate hexose1-phosphate transferases (Figure 2): in the succinoglycan biosynthesis pathway, ExoY transfers galactosyl 1-phosphate from UDP-galactose to und-P whereas synthesis of the xanthan repeat unit is started by GumD-catalyzed transfer of glucosyl 1phosphate from UDP-glucose to the lipid carrier. Serial activities of the glycosyltransferases ExoA, ExoL, ExoM, ExoO, and ExoU complete the synthesis of the succinoglycan repeat unit that is acetylated and succinylated to varying degrees by ExoZ and ExoH, respectively. While these modifications are not required for assembly of the polysaccharide, pyruvylation of the terminal glucose residue by ExoV seems to be essential for this process (Becker et al., 1993; Reuber and Walker, 1993). The xanthan repeat unit is completed by the glycosyltransferases GumM,
GumH, GumK, and GumI. Non-sugar decorations are added to the mannose residues of the repeat unit at varying degrees by the pyruvyltransferase GumL and the acetyltransferases GumF and GumG (Ielpi et al., 1993; Stankowski et al., 1993; Becker et al., 1998). Accumulation of intermediates carrying non-sugar substituents suggests that modification occurs at the level of repeat unit synthesis in the cytoplasm, before assembly to the polysaccharide (Ielpi et al., 1993; Reuber and Walker, 1993).

Und-PP-linked repeat units are then transported by the Wzx flippase to the periplasmic face of the inner membrane where they are polymerized to the polysaccharide by Wzy. In succinoglycan and xanthan biosynthesis, ExoT/ExoQ and GumJ/GumE represent the Wzx/Wzy proteins, respectively (González et al., 1998; Becker and Vorhölter, 2008). Wzx and Wzy protein sequences are poorly conserved in different bacteria which may reflect strict substrate specificities (Islam and Lam, 2013, 2014; Hong and Reeves, 2014). As revealed by topology mapping experiments, Wzx proteins typically contain 12 transmembrane helices (Mazur et al., 2005; Cunneen and Reeves, 2008; Islam et al., 2010; Marolda et al., 2010). They belong to the polysaccharide transporter (PST) family that is part of the multidrug/oligosaccharidyl-lipo/polysaccharide (MOP) exporter superfamily. Within this superfamily, the PST family is the most closely related to the multidrug and toxin extrusion (MATE) family of efflux proteins (Hvorup et al., 2003). Although a Xray crystal structure of a Wzx protein so far has not been established, a structure for the Pseudomonas aeruginosa Wzx protein was reported based on homology modeling using the MATE family protein NorM from Vibrio cholerae (He et al., 2010) as a suitable template, followed by genetic, bioinformatic, and biochemical structure validation (Islam et al., 2012). The structure model as well as data from site-directed mutagenesis suggest a cationic lumen which has a role in substrate binding during translocation and a cationic exit portal at the periplasmic face of the protein. MATE efflux proteins use proton- or sodiumcoupled antiport (Kuroda and Tsuchiya, 2009). Since protons were reported to affect gating of and be taken up by the $P$. aeruginosa Wzx protein, the flippase mechanism likely utilizes proton-mediated antiport (Islam et al., 2013a). Cross-species and cross-strain complementations of $w z x$ genes indicate that Wzx flippases demonstrate remarkable substrate specificity for their complete native repeat units (Hong et al., 2012; Wang et al., 2012; Hong and Reeves, 2014). Certain non-native repeat units can still be flipped, albeit very inefficiently, a limitation that can be partially compensated for through overexpression of the particular flippase; however, such non-native complementations have thus far only been shown to be successful when the flippase and the non-native repeat both originate from systems in which the und-PP-linked sugar is the same (Marolda et al., 2006; Hong and Reeves, 2014).

Wzy-dependent polymerization of und-PP-linked repeat units takes place in the periplasm. Wzy catalyzes transfer of the growing chain to the new und-PP-linked repeat unit resulting in growth of the polysaccharide chain at the reducing end (Robbins et al., 1967; Woodward et al., 2010). In this respect, Wzy has glycosyltransferase activity (Islam and Lam, 2014). The tertiary structure of Wzy proteins is unknown. Membrane topology 

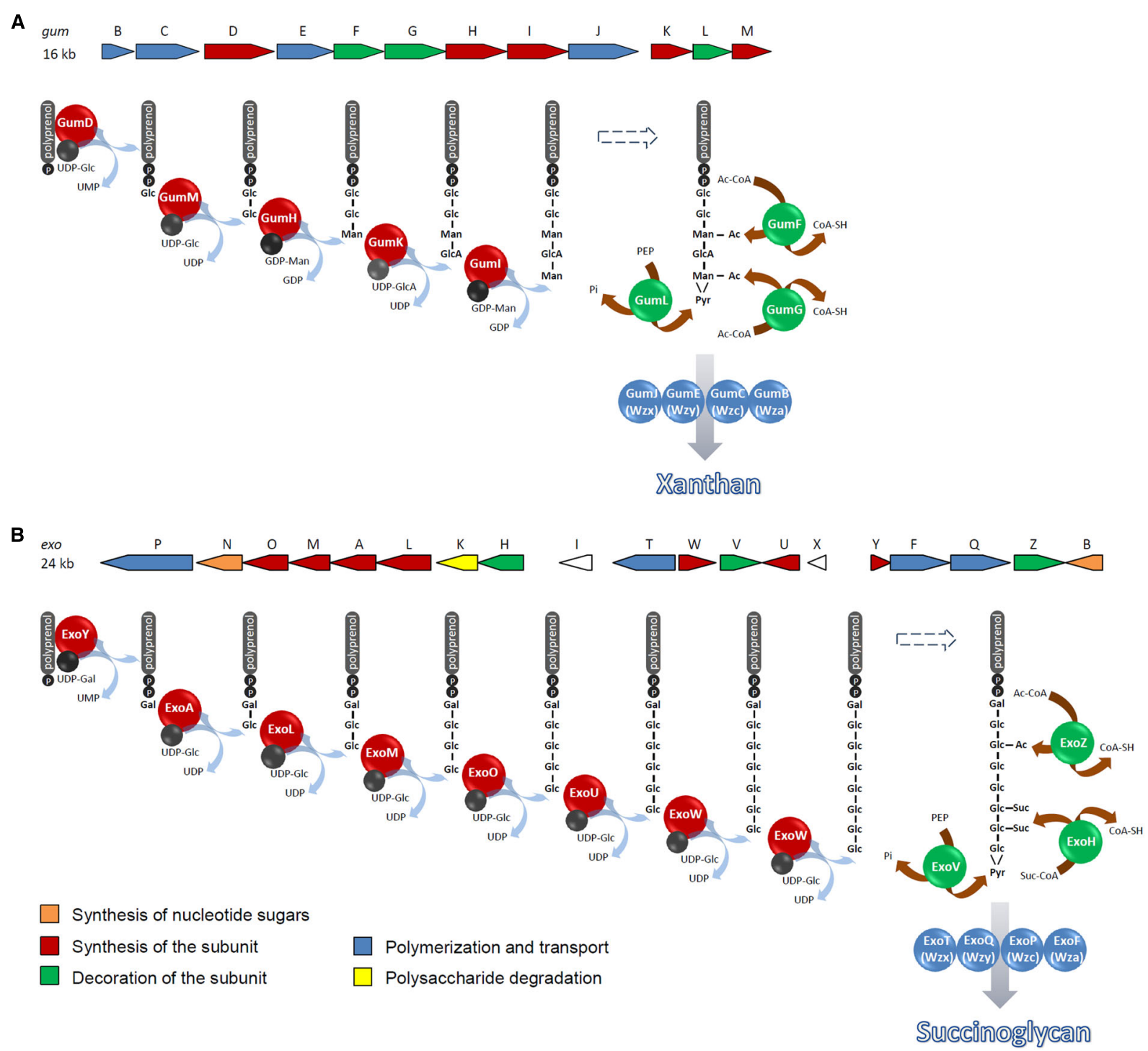

FIGURE 2 | Biosynthetic pathways of xanthan in X. campestris (A) and succinoglycan in S. meliloti (B). Repeat units are synthesized on a C55-undecaprenol phosphate lipid carrier at the inner face of the cytoplasmic membrane. Polymerization occurs by transfer of the lipid carrier-bound growing chain to a monomeric lipid carrier-bound repeat unit in the periplasm at the outer face of the cytoplasmic membrane. Modifications may occur during synthesis of the repeat unit before completion of the oligosaccharide. E.g., acetylated intermediates were detected in succinoglycan biosynthesis (Reuber and Walker, 1993). Ac, acetyl group; Ac-CoA, Acetyl-CoA; Gal, galactosyl group; Glc, glucosyl group; GlcA, glucuronyl group; Man, mannosyl group; PEP, phosphoenolpyruvate; Suc, succinyl group; Suc-CoA, succinyl-CoA. mapping indicates 12-14 transmembrane helices and two large periplasmic loops, the largest in the C-terminal and a smaller one in the N-terminal half (Daniels et al., 1998; Mazur et al., 2003; Islam et al., 2010). It is hypothesized that one of these loops binds the incoming und-PP-linked repeat unit while the other is binding the growing polysaccharide chain linked to und-PP (Islam et al., 2011, 2013b). A catch-and-release mechanism was proposed with one binding site responsible for recruiting the new subunit and the other binding site retaining the growing chain with a lower affinity that allows release and rebinding of the polysaccharide chain after each elongation step (Islam et al., 2011). Knowledge of the substrate specificity of Wzy proteins is very limited (Islam and Lam, 2014). Few examples have been reported for tolerance of Wzy proteins involved in O-antigen synthesis for differences in side-branch sugars and sensitivity for the repeat length of the main chain (Nurminen et al., 1971; Nyman et al., 1979; Reeves et al., 2013). In the biosynthetic pathway of succinoglycan and xanthan, ExoT/ExoQ and GumJ/GumE are the homologs of Wzx/Wzy (González et al., 1998; Becker and Vorhölter, 2008).

Transport through the periplasm and across the outer membrane is mediated by proteins of the PCP (polysaccharide copolymerase) and OPX (outer membrane polysaccharide export) families named $\mathrm{Wzz} / \mathrm{Wzc}$ and Wza, respectively. After the termination of polymerization for O-antigen chains (Daniels 
et al., 2002), the polysaccharide is transferred from the und-PP lipid carrier to the lipid A-core oligosaccharide acceptor by WaaL (Abeyrathne and Lam, 2007; Han et al., 2011; Ruan et al., 2012) to complete a molecule of LPS. Wzz proteins affect the length distribution of the O-antigen chain (Woodward et al., 2010). Although the sequence shows low conservation among different bacteria these integral inner membrane proteins of the polysaccharide copolymerase 1 (PCP-1) family have a characteristic topology with a large periplasmic domain flanked by an N-terminal and a C-terminal transmembrane helix (Morona et al., 1995; Whitfield et al., 1997). Oligomers of 5, 8, and 9 protomers were found analyzing X-ray crystal structures of different Wzz periplasmic domains (Tocilj et al., 2008; Kalynych et al., 2012). However, cryo-electron microscopy studies of full-length Wzz proteins indicated an invariant bell-shaped oligomer recently resolved as an octamer (Larue et al., 2009; Kalynych et al., 2015). Lack of Wzz results in unregulated Oantigen chains instead of the modal length of the $\mathrm{O}$-antigen chain characteristic to a specific LPS. Several models for Wzz protein function in chain-length regulation have been put forward (for a review, see Islam and Lam, 2014). The most recent, termed the "Chain-Feedback-Ruler" mechanism reconciles the majority of published data. It suggests that the growing chain is bound to the outer surface of the Wzz bell as soon as the oligosaccharide has reached the appropriate length for this binding. Interaction of Wzy, Wzz and the growing chain keeps the polymer in position for addition of further subunits. As the chain becomes longer it adopts higher-order structures that weaken the interaction with Wzz, which is thought to occur when the chain length exceeds the tip of the Wzz bell acting as a ruler. Through mechanical feedback this structural change is transmitted to the active site of Wzy resulting in release of the growing chain and ligation to the lipid A-core oligosaccharide.

Wzc proteins belong to the polysaccharide copolymerase $2 \mathrm{a}$ (PCP-2a) family engaged in assembly of high molecular weight CPS and EPS (Morona et al., 2000). These integral membrane proteins resemble $\mathrm{Wzz}$ proteins in their N-terminal half. This part represents a large periplasmic domain anchored in the inner membrane by two flanking transmembrane helices. The C-terminal part of Wzc proteins constitutes a cytosolic tyrosine autokinase domain transphosphorylating at multiple tyrosine residues (Cuthbertson et al., 2009). Wzc is a component of the trans-envelope polysaccharide translocation complex. Continued polymerization of repeat units requires $\mathrm{Wzc}$ and mutational studies imply that phosphorylation plays an important role in this process (Niemeyer and Becker, 2001; Paiment et al., 2002). In addition to Wzx, Wzy, and Wzc, capsule biosynthesis gene clusters frequently encode a tyrosine phosphatase (Wzb) which is likely involved in switching between phosphorylated and unphosphorylated states of Wzc (Wugeditsch et al., 2001; Cuthbertson et al., 2009). This cycling is thought to be important for polymerization of repeat units. Although, deletion of $w z c$ has a drastic effect on assembly of repeat units to the polymer, its exact role is still enigmatic. Models for the functional mechanism of Wzc propose a role as copolymerase that interacts with the polymerase Wzy or the initial glycosyltransferase to directly regulate polymerization or the supply of repeat units to the polymerization and export machinery (Cuthbertson et al., 2009). Another model proposes that Wzc oligomers serve as scaffold for organization of polysaccharide polymerization and export complexes (Cuthbertson et al., 2009). PCP-2a proteins required for polymerization of CPS and EPS differ from members of the PCP-1 family involved in O-antigen synthesis mainly by the presence of the kinase domain. Therefore, it was hypothesized that the kinase activity plays an important role particularly in biosynthesis of high molecular weight polysaccharides. In succinoglycan and xanthan biosynthesis ExoP and GumC are the Wzc homologs (Niemeyer and Becker, 2001; Becker and Vorhölter, 2008). In Sphingomonas elodea the periplasmic domain and the kinase domain of $\mathrm{Wzc}$ are encoded by two separate polypeptides, GelC and GelE (Moreira et al., 2005). Interestingly, GumC lacks the kinase domain and a kinase partner could not be identified in $X$. campestris (Cuthbertson et al., 2009) suggesting that this activity may not be required for synthesis of the high molecular weight EPS xanthan.

Wza is an OPX lipoprotein that was shown to interact with Wzc (Reid and Whitfield, 2005). While cryo-EM analysis suggested that Wzc forms tetramers, Wza forms octamers with a central channel through which the polysaccharide chain is transported across the outer membrane (Dong et al., 2006; Nickerson et al., 2014). Thus, both proteins build a machinery that accomplishes the transport of the polysaccharide from the periplasm to the cell surface (Collins et al., 2007). ExoF and GumB are the homologs of Wza in the biosynthetic pathway of succinoglycan and xanthan (Becker and Vorhölter, 2008; Cuthbertson et al., 2009).

\section{Challenges and Perspectives in Biosynthesis of Tailored EPS}

Exploiting the structural space of polysaccharides by combinatorial synthesis of novel EPS biosynthetic pathways is an attractive opportunity for synthetic biology. Major obstacles that need to be overcome on this route are substrate specificities at the levels of repeat unit biosynthesis, polymerization and export of polysaccharides that hinder free combination of biosynthetic components originating from different pathways. Failure to combine these components in one pathway may also arise from requirements for specific interactions of these proteins with other protein components of an EPS biosynthetic complex.

The CAZy (Carbohydrate Active Enzymes) database (Cantarel et al., 2009; Lombard et al., 2014) offers a wealth of information on glycosyltransferases. These enzymes catalyze the transfer of sugar moieties from activated donor molecules to specific acceptor molecules resulting in the formation of glycosidic bonds. During this reaction the stereochemistry of the substrate is either retained or inverted (Lairson et al., 2008). According to their domain structure, nucleotide sugar-dependent glycosyltransferases are classified into two major families. GT-A family enzymes comprise a single Rossman-like domain while GT-B enzymes contain two of these domains (Lairson et al., 2008; Cantarel et al., 2009). However, inverting and retaining enzymes are both present in the GT-A and GT-B families indicating that 
these different domain structures do not correlate with the catalytic mechanism (Lairson et al., 2008). Glycosyltransferases are further sub-classified according to catalytic activities and sequence similarities (Lombard et al., 2014). Nonetheless, for many subfamilies relationships between sequence and specificity are poorly understood impeding substrate predictions. Thus, enzymes with different substrate specificities frequently are members of the same family. Another problem arises from glycosyltransferases composed of several modules which may have different catalytic activities. Such proteins can be assigned to more than one family. The CAZy database is an invaluable resource for mining of glycosyltransferases to identify candidate enzymes for combinatorial synthesis of novel polysaccharides. Tens of thousands of potential glycosyltransferase genes have been revealed to be encoded in the numerous genomes and metagenomes that have been sequenced to date. Still, for the vast majority of these enzymes substrate and acceptor specificities are unknown. Future progress in linking sequence information to enzyme specificities, increasingly integrating protein structure information, will significantly accelerate the field. Yet, functionally important interactions of glycosyltransferases with other proteins of the EPS biosynthetic complex represent an additional gap of knowledge to be filled.

Furthermore, specific protein-protein interactions and substrate specificities strongly apply to assembly and export of the polymer. The low conservation of proteins involved in these terminal steps of EPS biosynthesis as well as swapping experiments of these components between different pathways imply substrate specificities related to the whole repeat unit structure. Only few examples of these components being conserved but structurally different polysaccharides being produced have been reported (Islam and Lam, 2014). Such cases may provide fundamental insights into the structural and mechanistic basis of substrate specificities.

\section{References}

Abeyrathne, P. D., and Lam, J. S. (2007). WaaL of Pseudomonas aeruginosa utilizes ATP in in vitro ligation of $\mathrm{O}$ antigen onto lipid A-core. Mol. Microbiol. 65, 1345-1359. doi: 10.1111/j.1365-2958.2007.05875.x

Aman, P., McNeil, M., Franzen, L., Darvill, A. G., and Albersheim, P. (1981). Structural elucidation using HPLC-MS and GLC-MS of the acidic polysaccharide secreted by Rhizobium meliloti strain 1021. Carbohydr. Res. 95, 263-282. doi: 10.1016/S0008-6215(00)85582-2

Becker, A., Katzen, F., Pühler, A., and Ielpi, L. (1998). Xanthan gum biosynthesis and application: a biochemical/genetic perspective. Appl. Microbiol. Biotechnol. 50, 145-152. doi: 10.1007/s002530051269

Becker, A., Kleickmann, A., Küster, H., Keller, M., Arnold, W., and Pühler, A. (1993). Analysis of the Rhizobium meliloti genes exoU, exoV, exoW, exoT and exoI involved in exopolysaccharide biosynthesis and nodule invasion: exoU and exoW probably encode glucosyltransferases. Mol. Plant Microbe Interact. 6, 735-744. doi: 10.1094/MPMI-6-735

Becker, A., and Pühler, A. (1998). "Production of exopolysaccharides," in The Rhizobiaceae: Molecular Biology of Model Plant-Associated Bacteria, eds H. P. Spaink, A. Kondorosi, and P. J. J. Hooykaas (Dordrecht: Kluwer Academic Publishers), 97-118.

Becker, A., and Vorhölter, F.-J. (2008). "Xanthan biosynthesis by Xanthomonas bacteria-an overview of the current biochemical and genomic data," in Microbial Production of Biopolymers and Polymer Precursors: Applications and Perspectives, ed. B. H. A. Rehm (Norfolk: Caister Academic Press), 1-11.
Based on the current state of knowledge ensuring the availability of precursors, such as nucleotide sugars, appears to be feasible. In contrast, more structural insights into the interactions of glycosyltransferases with the substrate and other components of the biosynthetic complex are required to chose the most promising candidates for combinatorial and directed evolution strategies toward novel or optimized specificities or for design-based engineering of these enzymes to function in a novel pathway. Even larger is the lack of fundamental knowledge of the terminal steps accomplished by the membrane protein complex that in Gram-negative bacteria spans the cytoplasmic membrane, the periplasm and the outer membrane. Recent progress in technologies that allow structural analysis of large membrane protein complexes, such as cryo-EM combined with data from crystal structure analysis, open new windows into a deeper mechanistic understanding of EPS polymerization and export. This would be crucial for succeeding in knowledge-based combinatorial assembly of novel functional EPS biosynthetic pathways in the future, particularly when components originating from different organisms are to be combined in one pathway. Yet another aspect that should receive consideration in pathway assembly as well as in replacement or addition of individual genes is clustering of biosynthetic genes which may promote membrane protein complex formation. Combinatorial assembly of novel pathways will also largely benefit from statistical design of experiments (DoE) strategies in bioengineering to meet unpredicted impacts of factor interactions and non-linear effects (Weissman and Anderson, 2014).

\section{Acknowledgment}

Work in the author's laboratory was funded by the LOEWE excellence program of the State of Hesse, Germany.

Cantarel, B. L., Coutinho, P. M., Rancurel, C., Bernard, T., Lombard, V., and Henrissat, B. (2009). The Carbohydrate-Active EnZymes database (CAZy): an expert resource for Glycogenomics. Nucleic Acids Res. 37, D233-D238. doi: 10.1093/nar/gkn663

Collins, R. F., Beis, K., Dong, C., Botting, C. H., McDonnell, C., Ford, R. C., et al. (2007). The 3D structure of a periplasm-spanning platform required for assembly of group 1 capsular polysaccharides in Escherichia coli. Proc. Natl. Acad. Sci. U.S.A. 4, 2390-2395. doi: 10.1073/pnas.0607763104

Cunneen, M. M., and Reeves, P. R. (2008). Membrane topology of the Salmonella enterica serovar Typhimurium Group B O-antigen translocase Wzx. FEMS Microbiol. Lett. 287, 76-84. doi: 10.1111/j.1574-6968.2008.01295.x

Cuthbertson, L., Mainprize, I. L., Naismith, J. H., and Whitfield, C. (2009). Pivotal roles of the outer membrane polysaccharide export and polysaccharide copolymerase protein families in export of extracellular polysaccharides in gram-negative bacteria. Microbiol. Mol. Biol. Rev. 73, 155-177. doi: 10.1128/MMBR.00024-08

Daniels, C., Griffiths, C., Cowles, B., and Lam, J. S. (2002). Pseudomonas aeruginosa $\mathrm{O}$-antigen chain length is determined before ligation to lipid A core. Environ. Microbiol. 4, 883-897. doi: 10.1046/j.1462-2920.2002.00288.x

Daniels, C., Vindurampulle, C., and Morona, R. (1998). Overexpression and topology of the Shigella flexneri O-antigen polymerase (Rfc/Wzy). Mol. Microbiol. 28, 1211-1222. doi: 10.1046/j.1365-2958.1998.00884.x

De, S. A. C., Sworn, G., and Iwata, R. (2015). Use of Succinoglycan in Frozen Food or Feedstuff Products, Patent WO2015014744 A1. Available at: http://www.google.co.in/patents/WO2015014744A1?cl=un 
Dong, C., Beis, K., Nesper, J., Brunkan-Lamontagne, A. L., Clarke, B. R., Whitfield, C., et al. (2006). Wza the translocon for E. coli capsular polysaccharides defines a new class of membrane protein. Nature 444, 226-229. doi: 10.1038/ nature 05267

Donot, F., Fontana, A., Baccou, J. C., and Schorr-Galindo, S. (2012). Microbial exopolysaccharides: main examples of synthesis, excretion, genetics and extraction. Carbohydr. Polym. 87, 951-962. doi: 10.1016/j.carbpol.2011. 08.083

Fink, J. K. (2003a). "Fluid loss additives," in Petroleum Engineer's Guide to Oil Field Chemicals and Fluids, ed. J. K. Fink (Oxford: Gulf Professional Publishing), 61-123.

Fink, J. K. (2003b). "Gelling agents," in Petroleum Engineer's Guide to Oil Field Chemicals and Fluids, ed. J. K. Fink (Oxford: Gulf Professional Publishing, Elsevier), 275-293.

Freitas, F., Alves, V. D., and Reis, M. A. (2011). Advances in bacterial exopolysaccharides: from production to biotechnological applications. Trends Biotechnol. 29, 388-398. doi: 10.1016/j.tibtech.2011.03.008

González, J. E., Semino, C. E., Wang, L. X., Castellano-Torres, L. E., and Walker, G. C. (1998). Biosynthetic control of molecular weight in the polymerization of the octasaccharide subunits of succinoglycan, a symbiotically important exopolysaccharide of Rhizobium meliloti. Proc. Natl. Acad. Sci. U.S.A. 95, 13477-13482. doi: 10.1073/pnas.95.23.13477

Greenfield, L. K., and Whitfield, C. (2012). Synthesis of lipopolysaccharide Oantigens by $\mathrm{ABC}$ transporter-dependent pathways. Carbohydr. Res. 356, 12-24. doi: 10.1016/j.carres.2012.02.027

Han, W., Wu, B., Li, L., Zhao, G., Woodward, R., Pettit, N., et al. (2011). Defining function of lipopolysaccharide $\mathrm{O}$-antigen ligase WaaL using chemoenzymatically synthesized substrates. J. Biol. Chem. 287, 5357-5365. doi: 10.1074/jbc.M111.308486

Harding, N. E., Raffo, S., Raimondi, A., Cleary, J. M., and Ielpi, L. (1993). Identification, genetic and biochemical analysis of genes involved in synthesis of sugar nucleotide precursors of xanthan gum. J. Gen. Microbiol. 139, 447-457. doi: 10.1099/00221287-139-3-447

He, X., Szewczyk, P., Karyakin, A., Evin, M., Hong, W. X., Zhang, Q., et al. (2010). Structure of a cation-bound multidrug and toxic compound extrusion transporter. Nature 467, 991-994. doi: 10.1038/nature09408

Hong, Y., Cunneen, M. M., and Reeves, P. R. (2012). The Wzx translocases for Salmonella enterica $\mathrm{O}$-antigen processing have unexpected serotype specificity. Mol. Microbiol. 84, 620-630. doi: 10.1111/j.1365-2958.2012. 08048.x

Hong, Y., and Reeves, P. R. (2014). Diversity of o-antigen repeat unit structures can account for the substantial sequence variation of wzx translocases. J. Bacteriol. 196, 1713-1722. doi: 10.1128/JB.01323-13

Hvorup, R. N., Winnen, B., Chang, A. B., Jiang, Y., Zhou, X. F., and Saier, M. H. Jr. (2003). The multidrug/oligosaccharidyl-lipid/polysaccharide (MOP) exporter superfamily. Eur. J. Biochem. 270, 799-813. doi: 10.1046/j.14321033.2003.03418.x

Ielpi, L., Couso, R. O., and Dankert, M. A. (1981). Pyruvic acid acetal residues are transferred from phosphoenolpyruvate to the pentasaccharide-PP-lipid. Biochem. Biophys. Res. Commun. 102, 1400-1408. doi: 10.1016/S0006291X(81)80167-2

Ielpi, L., Couso, R. O., and Dankert, M. A. (1983). Xanthan gum biosynthesis: acetylation accurs at the prenyl-phospho-sugar stage. Biochem. Int. 6, 323-333.

Ielpi, L., Couso, R. O., and Dankert, M. A. (1993). Sequential assembly and polymerization of the polyprenol-linked pentasaccharide repeating unit of the xanthan polysaccharide in Xanthomonas campestris. J. Bacteriol. 175, 2490-2500.

Islam, S. T., Eckford, P. D., Jones, M. L., Nugent, T., Bear, C. E., Vogel, C., et al. (2013a). Proton-dependent gating and proton uptake by Wzx support Oantigen-subunit antiport across the bacterial inner membrane. MBio 4, e0067813. doi: $10.1128 / \mathrm{mBio} .00678-13$

Islam, S. T., Huszczynski, S. M., Nugent, T., Gold, A. C., and Lam, J. S. (2013b). Conserved-residue mutations in Wzy affect O-antigen polymerization and Wzzmediated chain-length regulation in Pseudomonas aeruginosa PAO1. Sci. Rep. 3, 3441. doi: 10.1038/srep03441

Islam, S. T., Fieldhouse, R. J., Anderson, E. M., Taylor, V. L., Keates, R. A., Ford, R. C., et al. (2012). A cationic lumen in the Wzx flippase mediates anionic Oantigen subunit translocation in Pseudomonas aeruginosa PAO1. Mol. Microbiol. 84, 1165-1176. doi: 10.1111/j.1365-2958.2012.08084.x
Islam, S. T., Gold, A. C., Taylor, V. L., Anderson, E. M., Ford, R. C., and Lam, J. S. (2011). Dual conserved periplasmic loops possess essential charge characteristics that support a catch-and-release mechanism of O-antigen polymerization by Wzy in Pseudomonas aeruginosa PAO1. J. Biol. Chem. 286, 20600-20605. doi: 10.1074/jbc.C110.204651

Islam, S. T., and Lam, J. S. (2013). Wzx flippase-mediated membrane translocation of sugar polymer precursors in bacteria. Environ. Microbiol. 15, 1001-1015. doi: 10.1111/j.1462-2920.2012.02890.x

Islam, S. T., and Lam, J. S. (2014). Synthesis of bacterial polysaccharides via the Wzx/Wzy-dependent pathway. Can. J. Microbiol. 60, 697-716. doi: 10.1139/cjm2014-0595

Islam, S. T., Taylor, V. L., Qi, M., and Lam, J. S. (2010). Membrane topology mapping of the O-antigen flippase (Wzx), polymerase (Wzy), and ligase (WaaL) from Pseudomonas aeruginosa PAO1 reveals novel domain architectures. MBio 1, e00189-10. doi: $10.1128 / \mathrm{mBio} .00189-10$

Jansson, P. E., Kenne, L., and Lindberg, B. (1975). Structure of extracellular polysaccharide from Xanthomonas campestris. Carbohydr. Res. 45, 275-282. doi: 10.1016/S0008-6215(00)85885-1

Kalynych, S., Cherney, M., Bostina, M., Rouiller, I., and Cygler, M. (2015). Quaternary structure of WzzB and WzzE polysaccharide copolymerases. Protein Sci. 24, 58-69. doi: 10.1002/pro.2586

Kalynych, S., Yao, D., Magee, J., and Cygler, M. (2012). Structural characterization of closely related O-antigen lipopolysaccharide (LPS) chain length regulators. J. Biol. Chem. 287, 15696-15705. doi: 10.1074/jbc.M112.354837

Kuroda, T., and Tsuchiya, T. (2009). Multidrug efflux transporters in the MATE family. Biochim. Biophys. Acta. 1794, 763-768. doi: 10.1016/j.bbapap. 2008.11.012

Lairson, L. L., Henrissat, B., Davies, G. J., and Withers, S. G. (2008). Glycosyltransferases: structures, functions, and mechanisms. Annu. Rev. Biochem. 77, 521-555. doi: 10.1146/annurev.biochem.76.061005.092322

Larue, K., Kimber, M. S., Ford, R., and Whitfield, C. (2009). Biochemical and structural analysis of bacterial $\mathrm{O}$-antigen chain length regulator proteins reveals a conserved quaternary structure. J. Biol. Chem. 284, 7395-7403. doi: 10.1074/jbc.M809068200

Lee, K. Y., and Mooney, D. J. (2012). Alginate: properties and biomedical applications. Prog. Polym. Sci. 37, 106-126. doi: 10.1016/j.progpolymsci. 2011.06.003

Lombard, V., Golaconda Ramulu, H., Drula, E., Coutinho, P. M., and Henrissat, B. (2014). The carbohydrate-active enzymes database (CAZy) in 2013. Nucl. Acids Res. 42, D490-D495. doi: 10.1093/nar/gkt1178

Marolda, C. L., Li, B., Lung, M., Yang, M., Hanuszkiewicz, A., Rosales, A. R., et al. (2010). Membrane topology and identification of critical amino acid residues in the Wzx O-antigen translocase from Escherichia coli O157:H4. J. Bacteriol. 192, 6160-6171. doi: 10.1128/JB.00141-10

Marolda, C. L., Tatar, L. D., Alaimo, C., Aebi, M., and Valvano, M. A. (2006). Interplay of the Wzx translocase and the corresponding polymerase and chain length regulator proteins in the translocation and periplasmic assembly of lipopolysaccharide o antigen. J. Bacteriol. 188, 5124-5135. doi: 10.1128/JB.00461-06

Mazur, A., Krol, J. E., Marczak, M., and Skorupska, A. (2003). Membrane topology of PssT, the transmembrane protein component of the type I exopolysaccharide transport system in Rhizobium leguminosarum bv. trifolii strain TA1. J. Bacteriol. 185, 2503-2511. doi: 10.1128/jb.185.8.2503-2511.2003

Mazur, A., Marczak, M., Król, J. E., and Skorupska, A. (2005). Topological and transcriptional analysis of pssL gene product: a putative Wzx-like exopolysaccharide translocase in Rhizobium leguminosarum bv. trifolii TA1. Arch. Microbiol. 184, 1-10. doi: 10.1007/s00203-005-0018-Z

Melton, L. D., Mindt, L., Rees, D. A., and Sanderson, G. R. (1976). Covalent structure of the extracellular polysaccharide from Xanthomonas campestris: evidence from partial hydrolysis studies. Carbohydr. Res. 46, 245-257. doi: 10.1016/S00086215(00)84296-2

Moreira, L. M., Hoffmann, K., Albano, H., Becker, A., Niehaus, K., and Sá-Correia, I. (2005). Functional analysis of the gellan genes gelC and gelE from Sphingomonas elodea ATCC 31461 encoding two separate polypeptides homologous to the activator and to the kinase domains of phosphotyrosine autokinases. J. Mol. Microbiol. Biotechnol. 8, 43-57. doi: 10.1159/000082080

Morona, R., van den Bosch, L., and Daniels, C. (2000). Evaluation of Wzz/MPA1/MPA2 proteins based on the presence of coiled-coil regions. Microbiology 146, 1-4. doi: 10.1099/00221287-146-1-1 
Morona, R., van den Bosch, L., and Manning, P. A. (1995). Molecular, genetic, and topological characterization of $\mathrm{O}$-antigen chain length regulation in Shigella flexneri. J. Bacteriol. 177, 1059-1068.

Nickerson, N. N., Mainprize, I. L., Hampton, L., Jones, M. L., Naismith, J. H., and Whitfield, C. (2014). Trapped translocation intermediates establish the route for export of capsular polysaccharides across Escherichia coli outer membranes. Proc. Natl. Acad. Sci. U.S.A. 111, 8203-8208. doi: 10.1073/pnas.1400341111

Niemeyer, D., and Becker, A. (2001). The molecular weight distribution of succinoglycan produced by Sinorhizobium meliloti is influenced by specific phosphorylation and ATPase activity of the cytoplasmic domain of the ExoP protein. J. Bacteriol. 183, 5163-5170. doi: 10.1128/JB.183.17.5163-5170.2001

Nurminen, M., Hellerqvist, C. G., Valtonen, V. V., and Mäkelä, P. H. (1971). The smooth lipopolysaccharide character of 1,4,(5),12 and 1,9,12 transductants formed as hybrids between groups B and D of Salmonella. Eur. J. Biochem. 22, 500-505. doi: 10.1111/j.1432-1033.1971.tb01569.x

Nyman, K., Plosila, M., Howden, I., and Mäkelä, P. H. (1979). Genetic determination of lipopolysaccharide: locus of O-specific unit polymerase in group E of Salmonella. Zentralbl. Bakteriol. Orig. A 243, 355-362.

Osmalek, T., Froelich, A., and Tsarek, S. (2014). Application of gellan gum in pharmacy and medicine. Int. J. Pharm. 466, 328-340. doi: 10.1016/j.ijpharm.2014.03.038

Paiment, A., Hocking, J., and Whitfield, C. (2002). Impact of phosphorylation of specific residues in the tyrosine autokinase, $\mathrm{Wzc}$, on its activity in assembly of group 1 capsules in Escherichia coli. J. Bacteriol. 184, 6437-6447. doi: 10.1128/JB.184.23.6437-6447.2002

Paniagua-Michel Jde, J., Olmos-Soto, J., and Morales-Guerrero, E. R. (2014). Algal and microbial exopolysaccharides: new insights as biosurfactants and bioemulsifiers. Adv. Food Nutr. Res. 73, 221-257. doi: 10.1016/B978-0-12800268-1.00011-1

Reeves, P. R., Cunneen, M. M., Liu, B., and Wang, L. (2013). Genetics and evolution of the Salmonella galactose-initiated set of O antigens. PLOS ONE 8:e69306. doi: 10.1371/journal.pone.0069306

Rehm, B. H. A. (2009). Microbial Production of Biopolymers and Polymer Precursors: Applications and Perspectives. Norfolk: Caister Academic Press.

Reid, A. N., and Whitfield, C. (2005). Functional analysis of conserved gene products involved in assembly of Escherichia coli capsules and exopolysaccharides: evidence for molecular recognition between Wza and Wzc for colanic acid biosynthesis. J. Bacteriol. 187, 5470-5481. doi: 10.1128/JB.187.15.5470-5481.2005

Reinhold, B. B., Chan, S. Y., Reuber, T. L., Marra, A., Walker, G. C., and Reinhold, V. N. (1994). Detailed structural characterization of succinoglycan, the major exopolysaccharide of Rhizobium meliloti Rm1021. J. Bacteriol. 176, 1997-2002.

Reuber, T. L., and Walker, G. C. (1993). Biosynthesis of succinoglycan, a symbiotically important exopolysaccharide of Rhizobium meliloti. Cell 74, 269-280. doi: 10.1016/0092-8674(93)90418-P

Robbins, P. W., Bray, D., Dankert, M., and Wright, A. (1967). Direction of chain growth in polysaccharide synthesis: work on a bacterial polysaccharide suggests that elongation can occur at the "reducing" end of growing chains. Science 158, 1536-1542. doi: 10.1126/science.158.3808. 1536
Ruan, X., Loyola, D. E., Marolda, C. L., Perez-Donoso, J. M., and Valvano, M. A. (2012). The WaaL O-antigen lipopolysaccharide ligase has features in common with metal ion-independent inverting glycosyltransferases. Glycobiology 22, 288-299. doi: 10.1093/glycob/cwr150

Schmid, J., Sieber, V., and Rehm, B. (2015). Bacterial exopolysaccharides: biosynthesis pathways and engineering strategies. Front. Microbiol. 6:496. doi: 10.3389/fmicb.2015.00496

Stankowski, J. D., Mueller, B. E., and Zeller, S. G. (1993). Location of a second Oacetyl group in xanthan gum by the reductive-cleavage method. Carbohydr. Res. 241, 321-326. doi: 10.1016/0008-6215(93)80123-V

Tocilj, A., Munger, C., Proteau, A., Morona, R., Purins, L., Ajamian, E., et al. (2008). Bacterial polysaccharide co-polymerases share a common framework for control of polymer length. Nat. Struct. Mol. Biol. 15, 130-138. doi: 10.1038/nsmb.1374

Ullrich, M. (2009). Bacterial Polysaccharides: Current Innovations and Future Trends. Norfolk: Caister Academic Press.

Wang, X., Yang, F., and von Bodman, S. B. (2012). The genetic and structural basis of two distinct terminal side branch residues in stewartan and amylovoran exopolysaccharides and their potential role in host adaptation. Mol. Microbiol. 83, 195-207. doi: 10.1111/j.1365-2958.2011.07926.x

Weissman, S. A., and Anderson, N. G. (2014). Design of Experiments (DoE) and process optimization. a review of recent publications. Org. Process Res. Dev. doi: 10.1021/op500169m [Epub ahead of print]

Whitfield, C., Amor, P. A., and Köplin, R. (1997). Modulation of the surface architecture of gram-negative bacteria by the action of surface polymer:lipid Acore ligase and by determinants of polymer chain length. Mol. Microbiol. 23, 629-638. doi: 10.1046/j.1365-2958.1997.2571614.x

Whitney, J. C., and Howell, P. L. (2013). Synthase-dependent exopolysaccharide secretion in Gram-negative bacteria. Trends Microbiol. 21, 63-72. doi: 10.1016/j.tim.2012.10.001

Willis, L. M., and Whitfield, C. (2013). Structure, biosynthesis, and function of bacterial capsular polysaccharides synthesized by ABC transporter-dependent pathways. Carbohydr. Res. 378, 35-44. doi: 10.1016/j.carres.2013.05.007

Woodward, R., Yi, W., Li, L., Zhao, G., Eguchi, H., Sridhar, P. R., et al. (2010). In vitro bacterial polysaccharide biosynthesis: defining the functions of Wzy and Wzz. Nat. Chem. Biol. 6, 418-423. doi: 10.1038/nchembio.351

Wugeditsch, T., Paiment, A., Hocking, J., Drummelsmith, J., Forrester, C., and Whitfield, C. (2001). Phosphorylation of Wzc, a tyrosine autokinase, is essential for assembly of group 1 capsular polysaccharides in Escherichia coli. J. Biol. Chem. 276, 2361-2371. doi: 10.1074/jbc.M009092200

Conflict of Interest Statement: The author declares that the research was conducted in the absence of any commercial or financial relationships that could be construed as a potential conflict of interest.

Copyright (C) 2015 Becker. This is an open-access article distributed under the terms of the Creative Commons Attribution License (CC BY). The use, distribution or reproduction in other forums is permitted, provided the original author(s) or licensor are credited and that the original publication in this journal is cited, in accordance with accepted academic practice. No use, distribution or reproduction is permitted which does not comply with these terms. 